\title{
Cochrane and its prospects in Bosnia and Herzegovina: Relying on Cochrane Croatia
}

\author{
Mersiha Mahmić-Kaknjo ${ }^{1^{*}}$, Livia Puljak ${ }^{2}$, Filipa Markotić ${ }^{3}$, Mahir Fidahić ${ }^{4}$, \\ Lejla Muhamedagićs ${ }^{5}$ Irena Zakarija-Grković
}

${ }^{1}$ Department of Clinical Pharmacology Zenica Cantonal Hospital, Zenica, Bosnia and Herzegovina, ${ }^{2}$ Knowledge Translation Manager, Cochrane Croatia University of Split School of Medicine, Split, Croatia ${ }^{3}$ Centre for Clinical Pharmacology University Clinical Hospital Mostar Mostar, Bosnia and Herzegovina, ${ }^{4}$ Faculty of Medicine, University of Tuzla, Tuzla Bosnia and Herzegovina, ${ }^{5}$ Department of Ophthalmology, Public Institution Health Centre of Sarajevo Canton, Sarajevo Bosnia and Herzegovina, ${ }^{6} \mathrm{Co}$-Director Cochrane Croatia, University of Split School of Medicine, Split, Croatia

${ }^{*}$ Corresponding author:
mmahmickaknjo@gmail.com
Tel.: + 38732405133
Fax.: + 38732226576

Received: 5 February 2015

Accepted: 8 March 2015

Key words: Cochrane - Evidence-based Medicine $(\mathrm{EBM}) \cdot$ Croatia - Bosnia and Herzegovina
In this article we describe Cochrane and its products: Cochrane systematic reviews (CSRs) and other Cochrane evidence. Cochrane is a unique, international, non-profit organisation that offers health care providers, health care consumers and other decision makers unbiased and highly reliable information on health, which is pivotal for conscientious and responsible decision making in overall healthcare. Cochrane offers the highest ranked evidence in Evidence Based Medicine (EBM) - systematic reviews. Currently, CSRs are freely available in $\mathrm{BH}$, and therefore, they ought to be widely used, and understood. We will present the new Cochrane Strategy to 2020, which was the main topic of the $6^{\text {th }}$ Croatian Cochrane Symposium (CroCoS), as well as explore prospects for spreading Cochrane activities to Bosnia and Herzegovina $(\mathrm{BH})$, through collaboration with Cochrane Croatia. $\mathrm{BH}$ has no officially organized Cochrane activity, as yet. We hope that this article will raise awareness about Cochrane in $\mathrm{BH}$, help promote its activities, and deepen the existing collaboration with Cochrane Croatia. There are already some changes being introduced concerning Cochrane - at least, in one half, the Federation of $\mathrm{BH}(\mathrm{FBH})$. Two documents symbolising official recognition of policy changes towards Cochrane have recently been published in the Official Gazette of FBH. Conclusion. Since founding a BH Cochrane Branch would be costly and difficult to achieve in a complicated environment, such as the one we have, $\mathrm{BH}$ could use the good will, experience, knowledge, and translated educational, training and web materials of Cochrane Croatia, particularly given the language similarities, to promote evidence based medicine in $\mathrm{BH}$.

\section{Introduction}

The awareness of the importance of Evidence-based Medicine (EBM) and of Cochrane is growing in Bosnia and Herzegovina $(\mathrm{BH})$. However, $\mathrm{BH}$ does not have an official group of Cochrane or any organised Cochrane-related activities. In this article, we stress the importance of Cochrane's legacy and prospects for expanding its activities in $\mathrm{BH}$, with a particular focus on Cochrane Croatia, which was founded in 2008 and since then has become a strong partner to all stakeholders in that country - decision makers, physicians, and most recently, patients. Their knowledge, experience and good will towards collaboration ought to be used in $\mathrm{BH}$. For the time being, the most important step is to raise physicians' awareness of EBM and Cochrane in $\mathrm{BH}$. 
The highlights of the $6^{\text {th }}$ Annual Croatian Cochrane Symposium (CroCoS) will be presented in this paper as well. Finally, we will give a blueprint for the future of Cochrane activities in $\mathrm{BH}$.

\section{About Cochrane and Its Greatest Treasure: the Cochrane Database of Systematic Reviews (CDSR)}

Cochrane is a unique, international, nonprofit organisation that offers health care providers, health care consumers and other decision makers unbiased and highly reliable information on health, which is pivotal for conscientious and responsible decision making in overall healthcare.

Cochrane stands at the forefront of the EBM movement (1). As defined by Sackett et al., EBM is the "conscientious, explicit and judicious use of currently best evidence in making decisions about the care of individual patients and involves integrating individual clinical expertise with the best available clinical evidence and patient preferences"(2). The "best evidence" is generally accepted to be that arising from systematic reviews of randomised controlled trials (2), which is what Cochrane has been producing for over two decades.

Cochrane was founded as The Cochrane Collaboration in 1993 and named in honour of the British epidemiologist Archibald (Archie) Cochrane, who in 1979 wrote: "It is surely a great criticism of our profession that we have not organised a critical summary, by specialty or subspecialty, adapted periodically, of all relevant randomised controlled trials" (3). The Collaboration has grown enormously over the last two decades and in January this year it changed its name to 'Cochrane'. It is now a network of over 34,000 individuals, mostly volunteer authors, who work hard to produce systematic reviews (4). These individuals are supported by a network of Cochrane support groups, including centres, branches, review groups and fields. Cochrane's main product is the Cochrane Database of Systematic Reviews (CDSR), a central part of The Cochrane Library. The Cochrane Library is a collection of six databases that contain different types of high-quality, independent evidence to inform healthcare decision-making: Cochrane Database of Systematic Reviews (CDSR), Cochrane Central Register of Controlled Trials (CENTRAL), Cochrane Methodology Register (CMR),Database of Abstracts of Reviews of Effects (DARE), Health Technology Assessment Database (HTA), NHS Economic Evaluation Database (EED), and a seventh database that provides information about Cochrane groups, called About The Cochrane Collaboration. The Cochrane Library is a journal published by John Willey and Sons, with an impact factor in 2013 of 5.939 (5). The total number of citations received by the CDSR is the 6 th highest in the General \& Internal Medicine category of scientific journals. In 2013, only the top 3 ranked journals (NEJM, Lancet, $J A M A)$ received more citations than CDSR (6). CDSR Issue 11, published in 2014, comprises 6180 published reviews and 2352 protocols, totalling 8532 records (7), and reaching 8636 , the total number of records in 2015 (8).

Thanks to its middle income status, the whole territory of $\mathrm{BH}$ has one-click free access provided by Internet Protocol (IP) recognition (no individual login), meaning that all IP addresses from BH have unrestricted access to The Cochrane Library (9). Every user from $\mathrm{BH}$ can download the full text of every Cochrane Systematic Review (CSR) from the CDSR, which is a great convenience. This status is reviewed every January.

\section{Cochrane Systematic Reviews}

Systematic reviews synthesise all available clinical research on a particular topic, and therefore systematic reviews of randomised 
controlled trials (RCT) represent the highest level of evidence (10). They are the most rigorous way of determining whether a cause-effect relationship exists between an intervention and pre-determined outcomes (11). Double-blind placebo-controlled randomised trials (RCTs) are regarded as the gold standard tests in clinical research. Their strength lies in randomisation - random assignment to a study or a control group minimises all known and unknown factors that could influence the final results. In a systematic review, the data from more RCTs are combined, which increases the strength of evidence. Conducting a Cochrane Systematic Review (CSR) is a scientific investigation in itself, so-called secondary research, with the original studies being its "subjects" (12).

Each systematic review starts with a clinical question; the one proven to be still without a definite answer. After choosing the right question, a protocol is written. It is a peer-reviewed, predefined plan for conducting a review and it specifies the objectives, inclusion criteria, methods for study identification and selection, methods for assessing risk of bias in included studies, and outcomes of interest. Transparency and methodological rigour in producing a CSR are what makes them the 'gold standard' among systematic reviews. Since the protocol makes all these data available for public inspection, it functions as a public record explaining how the review authors intend to answer their research question (13).

After assessing for study validity, results of different independent studies are analysed in a process called a meta-analysis which represents a statistical summary of the results of the individual studies. Integrating numerical data from several primary studies that are related to the same question provides a more precise estimate of the intervention's effect. Yet, it is not always possible to conduct a meta-analysis in a systematic review, because if the study interventions or measured outcomes show considerable heterogeneity, a meta-analysis cannot be done (13).

There are five types of CSRs. The most common are intervention CSRs, which assess the benefits and harms of interventions used in healthcare and health policy. Methodology CSRs are prepared by a methodology group and address issues relevant to how systematic reviews and clinical trials are conducted and reported. The remaining types of CSRs are as follows: $d i-$ agnostic test accuracy reviews (aassess how well a diagnostic test performs in diagnosing and detecting a particular disease), qualitative reviews (synthesize qualitative evidence to address questions on aspects other than effectiveness), and prognosis reviews (address the probable course or future outcomes of people with a health problem) (14).

\section{Cochrane Croatia: involving physicians, medical students and patients}

With a goal to publicly advocate the use of Cochrane evidence in everyday health care decision making and to assist authors in producing CSRs, a Croatian branch of the Italian Cochrane Centre was established at the University of Split School of Medicine in 2008 (15). The Croatian Cochrane Branch is the reference centre for all surrounding countries, including $\mathrm{BH}$, which would like to promote EBM and become involved in the activities of Cochrane.

One of the first activities conducted by Cochrane Croatia was a nation-wide study among physicians in Croatia about their awareness and use of EBM databases and The Cochrane Library. This study indicated that a third of the respondents had heard about The Cochrane Library and 10\% of the respondents used the Library and read systematic reviews (16). In its conclusion the study highlighted that such a low level of awareness of EBM and The Cochrane Library warranted educational interven- 
tions for physicians, for the benefit of overall healthcare for patients in Croatia (16). Consequently, the majority of initiatives initially undertaken by Cochrane Croatia were focused on healthcare workers, mostly physicians. The first Croatian Cochrane Symposium was organised in 2009 , and the same year a regular column presenting CSRs started appearing in the Medical Gazette, the official journal of Croatian Medical Chamber. Various workshops and lectures for physicians were organized in hospitals and during symposia.

Another significant effort has been made in raising awareness of EBM and Cochrane in Croatia, this time among medical and health sciences students. In 2010 the Medical School in Split introduced a mandatory, vertically integrated course in research methodology into medical and dental curricula. The requests for curricular changes issued by the European Commission were answered by shaping the Split curricula into an original blend of traditional pre-clinical and clinical subjects with several vertically integrated subjects focusing on mastering clinical skills, professional attitudes, information management and critical, as well as evidence-based reasoning and decision making (17). Marušić et al. have found that the new course succeeded in increasing students' knowledge and skills for critical thinking and EBM, and prepared them for life-long learning in medicine (18).

Students at the University of Split School of Medicine are taught EBM from the first day of their studies, but there is a need to enhance their skills in evidence-searching and critical appraisal of the evidence in clinical practice, with real patients. As revealed by Vrdoljak et al. (19), teaching final-year students the practical use of EBM in a general practitioner's office might play an important role in the students' professional development. It may positively influence the quality of their future work, making EBM a basis for their life-long learning.

Practical Evidence About/For Real Life Situations (PEARLS), published by Cochrane Primary Health Field, are intended to make decision making even faster, easier and more adjusted to suit the needs of primary care practitioners. PEARLS have been regularly translated by members and collaborators of Cochrane Croatia since 2013.

However, the growing awareness of a need to shift focus from health care professionals to the lay consumers of evidence has been recognised by Cochrane Croatia as well. Patients should be involved in the decision-making process as equal partners. They should be able to access and get high-quality information about health in general and medical evidence should serve them directly. Therefore, since 2012 several initiatives have already been implemented in order to engage consumers in the activities of Cochrane Croatia. This idea was supported at the national level by Croatian Ministry of Science, Education and Sports, which awarded five grants to Cochrane Croatia for the "popularisation of science". Three of them have been used for engaging patient groups in selecting and translating Plain Language Summaries (PLSs) of CSRs into Croatian. A PLS is an overview of the findings of a Cochrane Systematic Review written in a language appropriate for patients and family members. The summary contains a brief statement of the significance of the review, the main findings of the professional review, preferably including the number of studies evaluated and number of total participants, comments on any adverse effects and limitations of the review. Thus, in 2012, Croatian became the sixth language in the world, after Spanish, French, Portuguese, Chinese and Arabic, in which translated PLS were made available. Croatian PLSs are published on the Croatian version of the Cochrane web site: http://www. cochrane.org/hr/health-evidence. 
Soon after, Cochrane Croatia recognised the increasingly important role of social networks in Croatian society and their potential in disseminating Cochrane evidence and other EBM-related information. As a result, in March 2013 a Facebook page called "Croatian Cochrane Branch" was opened and it currently has 1,902 "likes" (as of February 26, 2015).

One grant for the popularisation of science was invested in translation of the book "Testing Treatments", written by Imogen Evans, Hazel Thornton, Iain Chalmers and Paul Glasziou. The book-related website 'Testing Treatments Interactive' has also been translated into Croatian and offers its visitors an opportunity to read the book free of charge at http://hr.testingtreatments.org/ procitajte-knjigu-gdje-su-dokazi/.

Finally, since it has become clear that more emphasis should be put on education of the lay audience on the uselessness of many alternative and complementary therapies that are offered by 'quacks' in the market, the fifth grant for popularization of science is being used for the development of a website called "Evidence in medicine"(http:// dokaziumedicini.hr/), where anybody can ask questions about effectiveness and safety of interventions in medicine, and a group of scientists respond to these questions based on the best evidence available.

In September 2014, a nationwide survey of the knowledge and usage of EBM and CSRs was conducted among Croatian urban and rural patients visiting their family doctors on a regular basis, and its results are expected to be available in 2015. The survey results will show whether educational initiatives conducted by Cochrane Croatia have reached their intended consumer audience, and will provide guidance for the future work aimed towards consumers of Cochrane evidence.

The Croatian Cochrane Symposium (CroCoS) is traditionally held at the Medical School in Split, every year in spring time.
The $6^{\text {th }}$ CroCoS was held on Friday 6th June 2014, on the topic 'Better Evidence for a Better Future: Cochrane's Strategy to 2020' and it consisted of eight presentations, two parallel round tables and two concurrent workshops for future/current Cochrane authors.

Mark Wilson, Chief Executive Officer (CEO) of Cochrane, spoke on the organization's current position and strategic plans, emphasizing the fact that Cochrane is a unique, innovative and global independent network of evidence-based medicine enthusiasts and a recognised leader in its field, with a global reputation for quality and integrity. However, he also said it is necessary to transform Cochrane into a more effective and sustainable organisation in order for it to achieve its long-term goals. Activities of Cochrane must be focused on advocating high-quality, relevant and up-to-date evidence that will be useful and accessible to everybody, everywhere in the world, to enable making informed decisions in health care.

Dr. Irena Zakarija-Grković, Co-Director of Cochrane Croatia, spoke about the power of partnerships built within the Croatian Cochrane Network. This network has already included five professional societies and five patients' associations as official partners. One of the professional associations is the Medical Chamber of Zenica-Doboj Canton from $\mathrm{BH}$. The aim of the network is to connect with various partner institutions, professional societies and patient organisations which are interested in promoting evidencebased medicine.

Professor Ana Marušić, the Research Coordinator of Cochrane Croatia, spoke about different ways of using Cochrane evidence to create basic national drug lists. She presented the results of a study in which the Croatian Basic List of Medicines and the World Health Organization Model List of Essential Medicines (WHO EML) were compared. This study found that there are medicines on the Croatian Basic List of Medicines which 
were rejected by WHO's EML Committee, mostly due to lack of evidence for their effectiveness (20). In this study they used CSRs as surrogate markers of drug effectiveness. Professor Marušić also presented the calculated costs of using the drugs which have been rejected by the WHO.

\section{Cochrane's Coming of Age}

In March 2013, more than 200 Cochrane leaders from all over the world met in $\mathrm{Ox}$ ford, UK, to commemorate the first 20 years of the Cochrane Collaboration and to work together on a strategic framework for the next several years. This task, led by Cochrane's CEO, Mark Wilson, incorporated an analysis of the organisation's current strategic framework, a series of policy and strategy documents developed by Cochrane contributors from a 2008-9 Strategic Review, and wide consultations and feedback from contributors. A first draft of the new Cochrane Strategy was released for contributors and selected external stakeholders in July 2013, and following extensive feedback and numerous meetings, a final draft was presented and accepted by the organisation's members at the 2013 Annual General Meeting, on $21^{\text {st }}$ September, in Quebec City, Canada, at the $21^{\text {st }}$ Cochrane Colloquium.

The Cochrane Collaboration's first 20 years have been an astonishing success, with Cochrane significantly contributing to the mainstream acceptance of "Evidence-Based Medicine", and becoming the acknowledged, global leader in systematic review production. In 2008, there were fewer than 10,000 authors; today there are more than 22,400 active registered authors of CSRs, including those 42 from Croatia. There has been dramatic growth in the organisational infrastructure with currently 14 Regional Centres and 24 Branches (including the Croatian Cochrane Branch), 53 Review Groups, 16 Methods Groups and 12 thematic Fields and Networks. With the increasing number of authors, Cochrane review production has grown exponentially over the years, with more than 8,100 full CSRs and Protocols in the CDSR. The rising awareness and use of Cochrane evidence has followed, with over 7.4 million full-text downloads and 13.5 million abstract page views in 2013. In addition, another 4,181,131 million page views of Cochrane Summaries were recorded that same year (21).

Throughout this journey, Cochrane has had to face numerous challenges. These included managing the complex CSR production process, or more specifically, prioritising reviews and updates of reviews, training authors, providing review groups with adequate support, facing increasingly long review production processes, maintaining the quality of reviews and stimulating authors to complete and update reviews. Other constant challenges are decreasing the knowledge translation gap, improving the "User Experience", allowing global access to Cochrane content, securing funds for Cochrane activities, involving contributors from non-English speaking and low and middle income countries and facing rising competition. Cochrane's response to these challenges is its Strategy to 2020.

\section{Cochrane's Strategy to 2020}

The four main goals in Strategy to 2020 represent the desired endpoints for achieving the organisation's mission (22). Specific objectives describing the ways in which goals will be achieved and activities conducted have been set up for each goal.

Goal one is to produce evidence faster and more effectively, without compromising on quality. The objectives include quality assurance, relevance, timeliness, wide coverage, pioneering methods and efficient production. In 2014 alone, SMART (Specific, Measurable, Attainable, Relevant and Time- 
Bound) targets for goal one were set up in order to develop a list of approximately 200 new "high-priority" and "up-to-date" CSRs (that was published in January 2015 on the Cochrane.org website), as well as to improve production processes by implementing an author support tool and establishing a strategy for reducing review production time by 30 percent.

Goal two focuses on the users of Cochrane evidence, with the aim of making Cochrane content accessible to everyone, anytime and anywhere in the world, in a format that is user-centred. Cochrane will strive for a greater understanding of the end-users' experience with and their actual need for Cochrane evidence. A comprehensive translation strategy is already underway, aiming to make as much Cochrane content available in as many languages as possible, with at least key content translated into all WHO official languages by 2020 the latest. Cochrane Croatia is also playing a small but significant role in the translation of Cochrane PLSs and Cochrane launched a Croatian-language version of its Cochrane. org website in 2015. With the support of the Croatian Ministry of Science, Education and Sports, Cochrane Croatia, together with its growing team of collaborators, has translated over 700 PLSs, making quality evidence accessible to all citizens of Croatia and other neighbouring countries. Currently, full texts of CSRs are available only 12 months after their publication and by 2020 Cochrane aims to make all of its reviews available. Once a review is published, it risks getting forgotten if not properly disseminated, and because of that Cochrane has already incorporated in its Strategy various plans on how to build a dissemination framework into editorial process of CSRs to ensure that every review gets its own dissemination plan created to meet the needs of the targeted users.

Goal three of the Strategy is set up to make Cochrane a recognisable "go to" place for EBM. This will be achieved by creating an overarching, recognisable "Cochrane" brand; building greater recognition of the role of Cochrane as an essential link between primary research and health decisionmaking; advocating for evidence-informed health care and the uptake of synthesized research evidence in health policy making and services planning; promoting high-quality primary research which answers real world health questions and improves the evidence base upon which CSRs are built; by campaigning for transparency and integrity in scientific conduct; and by building international and local partnerships.

Finally, for all of these ambitious plans to be achieved, it is required to have an effective and sustainable organisation (Goal 4). Cochrane is committed to being inclusive and open, global and diverse, financially strong, efficiently run, investing in people, transparently governed and environmentally responsible. It is also planning to introduce a membership scheme in order to improve its organisational cohesiveness and reduce possible barriers to participation. A training and professional development strategy is underway, as well as a review of Cochrane's governance structure and processes. Strategy to 2020 encompasses a program on how to identify, mentor and train future leaders of the organisation. Cochrane is rapidly increasing the number of women in leadership positions: both of its Co-Chairs of the organization's Steering Group (Governing Board) are female as are over $60 \%$ of the present Steering Group. It is also committed to increasing diversity in other ways, to have more than $50 \%$ of its leaders originating from non-English speaking countries by 2020 .

Strategy to 2020 is an opportunity to create a world of improved health by producing and promoting use of high-quality evidence informed research, and then making its results usable and accessible to anyone who needs it. Through collaboration with $\mathrm{Co}-$ 
chrane Croatia, $\mathrm{BH}$ researches, academics, health professionals and others involved in health research have an opportunity to join Cochrane on this exciting journey.

\section{Prospects of Cochrane in $\mathrm{BH}-$ "Cochranising" BH: A wave from the city of Diocletian}

In order to analyse prospects of Cochrane in $\mathrm{BH}$, one first has to understand $\mathrm{BH}$ 's unique political situation. BH comprises two autonomous entities: the FBH and Republic of Srpska, with another region, the Brčko District $\mathrm{BH}$, governed by local authorities. Saying that $\mathrm{BH}$ is decentralised would be a large understatement. In $\mathrm{BH}$, there are less than 4 million people (23) and furthermore, 4 levels of power (municipal, cantonal, federal and state) with as many institutions governing. In such a decentralised environment, trying to organise any kind of institutional Cochrane entity is almost impossible. Is it necessary at all?

During the 6th CroCoS, Mark Wilson, Cochrane's CEO, stressed that the organization was committed to establishing flexible structures that best meet the needs and contexts of different countries, regions and parts of the world. He suggested that networks based on multiple hubs of people working on Cochrane activities in different places may work well for $\mathrm{BH}$ and the wider region. Founding an official entity in every country is a huge burden for any organisation, especially for those that operate and rely primarily on voluntary efforts.

When it comes to $\mathrm{BH}$, there are already some changes being introduced concerning Cochrane - at least, in one half, the FBH. Two documents symbolising official recognition of policy changes towards Cochrane have recently been published in the Official Gazette of FBH. The first document was published on $7^{\text {th }}$ July 2014, only a month after the $6^{\text {th }}$ CroCoS was held (24). In it, the ability of proper use of The Cochrane Library was listed as one of the essential skills required for clinical pharmacology trainees to have developed in their training program curriculum. The second important document was also published in the Official $\mathrm{Ga}$ zette of $\mathrm{FBH}$ on $15^{\text {th }}$ October 2014, in which The Cochrane Library was classified as an officially acknowledged source of information for another emerging issue in $\mathrm{BH}$ : Health Technology Assessment (HTA) (25).

In Croatia, there are many good examples of how to raise awareness and enhance the usage of reviews offered by Cochrane that should be followed. In ZenicaDoboj Canton, there is already research being conducted on evaluating attitudes and awareness of physicians towards EBM and Cochrane. Also, it is important that Cochrane's strict methodology is recognised as the "gold standard" in conducting systematic reviews: the first $\mathrm{BH}$ authors to use Cochrane methodology in performing a systematic review were Salkić at al. (26), although the review was not published in Cochrane Library. Another important step is to gather all Cochrane sympathisers in $\mathrm{BH}$. We could again follow the example of Cochrane Croatia in its use of social media in order to reach the general population. It would be a very useful yet inexpensive way to reach as many health care professionals and health care users as possible, but some kind of organised approach will be needed, without any doubt. It would be helpful if an institution could be found to provide at least a part time administrator, educator, health professional or researcher - a person and a place to gather and distribute information on Cochrane's $\mathrm{BH}$ activities.

Integrating a mandatory course on research methodology into medical schools' curriculum is of primary importance. Integrating Cochrane evidence such as Cochrane Clinical Answers into GPs' education is highly desirable. In order to over- 
come the language barrier when using English sources, which is a serious problem for most senior physicians, multicultural and multilingual $\mathrm{BH}$ should again rely on collaboration with its neighbouring countries. As mentioned above, Cochrane Croatia is currently conducting a translation project, financed by government grants and aimed at translating Cochrane PLSs, among other materials, into Croatian. Croatian is one of three official languages in $\mathrm{BH}$, and the differences between Croatian, on the one hand, and Bosnian and Serbian, on the other, are not an obstacle for understanding abstracts in Croatian by people speaking these other two constitutive $\mathrm{BH}$ languages. Translated PLSs could serve patients and their health care providers. Other steps in collaborating with Cochrane Croatia have also been undertaken. The Medical Chamber of ZenicaDoboj Canton has become an official partner of Cochrane Croatia; the Chamber representatives attended the first introductory and priority setting meeting for Cochrane partners at this year's Croatian Cochrane Symposium. We believe that this step could be the first in making a Cochrane nucleus in $\mathrm{BH}$. In addition, we have arranged everything to make the online course on systematic reviews, translated and prepared by Cochrane Croatia, available to all $\mathrm{BH}$ doctors, with CME points included.

Let us collaborate together and overcome all the boundaries that politics have forced upon us (ethnic, territorial, organisational) so that we can create a society in which patients and health care users are enabled to access high-quality, evidence-based information on health interventions. Last, but not least, we should aim to overcome barriers largely nourished by us, in the form of inter-specialty intolerance. Together, as citizens dedicated to EBM, we can change our world for the better.

\section{Conclusion}

Awareness of the importance of Cochrane, a synonym for evidence-based health care- is emerging in $\mathrm{BH}$. Croatian Cochrane is pivotal for spreading Cochrane activities in $\mathrm{BH}$. $\mathrm{BH}$ could benefit from using Cochrane Croatia's experience, good will and translated materials to establish Cochrane's position in $\mathrm{BH}$. There is a need for a Cochrane Hub in $\mathrm{BH}$.

\section{What is already known on this topic}

- Cochrane (formerly The Cochrane Collaboration) was founded in 1993. Cochrane Croatia, a branch of the Italian Cochrane Centre, was founded in 2008 as a voluntary, non-profit organisation based at the University of Split School of Medicine as part of the Croatian Centre for Global Health. Its main roles are production of systematic reviews, promotion of evidence-based medicine and dissemination of Cochrane evidence throughout South East Europe.

\section{What this study adds}

- This paper presents the new Cochrane Strategy to 2020, and highlights from the 6th Croatian Cochrane Symposium (CroCoS). Also, in this paper we propose a blueprint for future as Cochrane-related activities in Bosnia and Herzegovina.

Acknowledgements: Authors thank Ana and Matko Marušić for their unconditional support in writing this manuscript. We are grateful to Merima Mahmić for paper proofreading. We would also like to thank Mark G. Wilson, Dario Sambunjak and Davorka Vrdoljak for critically reviewing this article.

Authors' contributions: Conception and design: MMK; Acquisition of data, or analysis and interpretation of data: MMK, LP, FM, MF, LM, IZG; Drafting the article or revising it critically for important intellectual content: MMK, LP, FM, MF, LM, IZG; Final approval of the version to be published: MMK, LP, FM, MF, LM, IZG.

Conflict of interest: The authors declare that they have no conflict of interest.

\section{References}

1. Shah HM, Chung KC. Archie Cochrane and his vision for evidence-based medicine. Plast Reconstr Surg. 2009;124(3):982-8. 
2. Sackett DL, Rosenberg WM, Gray JA, Haynes RB, Richardson WS. Evidence based medicine: what it is and what it isn't. BMJ. 1996;312(7023):71-2.

3. Cochrane AL. 1931-1971: a critical review, with particular reference to the medical profession. Medicines for the year 2000. London: Office of Health Economics; 1979. p. 1-11.

4. Allen C, Richmond K. The Cochrane Collaboration: international activity within Cochrane Review Groups in the first decade of the twenty-first century. J Evid Based Med. 2011;4(1):2-7.

5. The Cochrane Collaboration. Cochrane Database of Systematic Reviews (CDSR). 2014 [cited 2014 November 30]. Available from: http://www.cochrane. org/editorial-and-publishing-policy-resource/cochrane-database-systematic-reviews-cdsr.

6. The Cochrane Collaboration. The Cochrane Library 2013 Impact Factor and usage report. 2013 [cited 2014 November 30]. Available from: www. cochrane.org/sites/default/files/uploads/EPPR/ CDSR_Impact-factor_and-usage-report_2013.pdf.

7. The Cochrane Community (beta). Cochrane Database of Systematic Reviews in numbers. 2014 [cited 2015 March 5]. Available from: http:// community.cochrane.org/cochrane-reviews/cochrane-database-systematic-reviews-numbers.

8. Cochrane Library. Cochrane Database of Systematic Reviews. 2015 [cited 2015 March 5]. Available from: http://www.cochranelibrary.com/cochranedatabase-of-systematic-reviews/index.html.

9. The Cochrane Collaboration. Access options for the Cochrane Library. [cited 2014 November 30]. Available from: www.thecochranelibrary.com/ view/0/FreeAccess.html.

10. Evans D. Hierarchy of evidence: a framework for ranking evidence evaluating healthcare interventions. J Clin Nurs. 2003;12(1):77-84.

11. Sibbald B, Roland M. Understanding controlled trials. Why are randomised controlled trials important? BMJ. 1998;316(7126):201.

12. The Cochrane Collaboration. Cochrane Reviews. [cited 2014 December 17]. Available from: http:// www.cochrane.org/cochrane-reviews.

13. The Cochrane Collaboration. The Cochrane Library-About Cochrane Systematic Reviews and Protocols. [cited 2014 December 17]. Available from: http://www.thecochranelibrary.com/ view/0/AboutCochraneSystematicReviews.html.

14. Cochrane Library. About Cochrane Reviews. 2015 [cited 2015 March 5]. Available from: http://www. cochranelibrary.com/about/about-cochrane-systematic-reviews.html.

15. Puljak L, Rako D. Branch of the Cochrane Collaboration founded in Croatia. Acta Med Acad. 2009;38:51-4.
16. Novak K, Miric D, Jurin A, Vukojevic K, Aljinovic J, Caric A, et al. Awareness and use of evidencebased medicine databases and Cochrane Library among physicians in Croatia. Croat Med J. 2010;51(2):157-64.

17. Grkovic I, Sapunar D, Marusic M. Ways to address the challenges of a modern medical curriculum: living academic medicine at the University of Split, School of Medicine. Acta Med Acad. 2012;41(1):7-17.

18. Marusic A, Malicki M, Sambunjak D, Jeroncic A, Marusic M. Teaching science throughout the sixyear medical curriculum: two-year experience from the University of Split School of Medicine, Split, Croatia. Acta Med Acad. 2014;43(1):50-62.

19. Vrdoljak D. Teaching evidence based medicine in family medicine. Acta Med Acad. 2012;41(1):88-92.

20. Jelicic Kadic A, Zanic M, Skaricic N, Marusic A. Using the WHO Essential Medicines List to Assess the Appropriateness of Insurance Coverage Decisions: A Case Study of the Croatian National Medicine Reimbursement List. PLoS One. 2014;9(10):e111474.

21. Wilson M. Cochrane: Continuing Global Leadership in Evidence-Based Medicine. 2014 [cited 2014 December 8]. Available from: http://news. fm.ul.pt/Backoffice/UserFiles/File/News\%2042/ Cochrane\%20-\%20Portuguese $\% 20 \mathrm{Branch} \% 20$ Opening\%20-Mark\%20Wilson.pdf.

22. The Cochrane Collaboration. Strategy to 2020. 2013 [cited 2015 March 5]. Available from: http://community.cochrane.org/sites/default/files/uploads/ Cochrane_Strategy\%20to\%202020_Final\%20public\%20access\%20version_corrected.pdf.

23. Agency for statistics of Bosnia and Herzegovina. Preliminary results of the 2013 Census of Population, Households and Dwellings in Bosnia and Herzegovina. 2013 [cited 2014 November 30]. Available from: www.bhas.ba/obavjestenja/Preliminarni_rezultati_bos.pdf. .

24. Clinical Pharmacology with toxicology. Official Gazette of BH Federation, No 54/14, Pg 92. 2014.

25. Rule book on the principles of introducing new health technologies in public health institutions, as well as in private health praxis, and the procedure on giving permission for using health technologies. Official Gazette of BH Federation, No 84/14, Pg 67. 2014.

26. Salkic NN, Jovanovic P, Hauser G, Brcic M. FibroTest/Fibrosure for significant liver fibrosis and cirrhosis in chronic hepatitis B: a meta-analysis. Am J Gastroenterol. 2014;109(6):796-809. 